\title{
Stress Calculations Adopted Finite Element Method of Pressure Steam Pipeline Containing Defects
}

\author{
Yonggui Chen ${ }^{1,2}$, Ping Tang ${ }^{1,2, *}$, Jiang Zhong ${ }^{3}$, Sunting Yan ${ }^{1,2}$, Nanhui Jin ${ }^{1,2}$, Lintao Liu ${ }^{3}$ \\ ${ }^{1}$ Zhejiang Academy of Special Equipment Science, Hangzhou 310020, China \\ ${ }^{2}$ Key Laboratory of Special Equipment Safety Testing Technology of Zhejiang Province, Hangzhou 310020, China \\ ${ }^{3}$ Tongxiang Ties Environmental Energy co., Ltd., China
}

\begin{abstract}
The stress behavior of defects is the key factor in the safety assessment of pressure steam pipeline containing defects. For achieving carbon peaking and carbon neutrality, high-parameter pressure steam pipelines are widely designed in the recent years. It makes the safety assessment based on defect stresses become significantly important. A combined FEA method of stress calculations was proposed in the present study for calculating film stress and bend stress of defects located in a pressure steam pipeline from a certain power plant. An efficient program code with graphical user interface was designed to automatically generate FEA models of defect part pipelines. It is truly an innovative highlight of the present work attributed to convenient operation and outstanding efficiency. This paper provides an available way to obtain the stress state of defects in the safety assessment of pressure steam pipeline containing defects.
\end{abstract}

Key Words. Pressure steam pipeline, Large-scale defects, Stress state, Structural integrity.

\section{Introduction}

Pressure steam pipeline is general equipment in petrochemical and power industry. The failure of its structural integrity may result in costly financial losss, severe environmental harms and catastrophic accidents of many casualties[1]. With the development trend of achieving carbon peaking and carbon neutrality through energy saving, emission reduction, and improvement of energy efficiency ratio in various countries around the world, an amount of high-parameter steam pipeline are widely designed, whose safe operateion is mostly threatened by pipeline defects associated with mechanical damage. Such high-temperature and high-pressure working environment may more rapidly lead to plastic failure of pipeline defects [2-3]. Therefore, the safety assessment of pressure steam pipeline containing defects based on actual defect stresses has become an increasingly urgent issue[4-6].

However, it is not easy to get the magnitude and distribution of defect stresses of pressure pipelines. Experimental measurement is hard and costly although as the mostly direct way. The complicated factors including pipeline structure, pipeline layout, support constraints and external loads determine that accurate theoretical calculations of defect stresses are difficult to accomplish[7]. Besides, The huge capacity of 3D shell element models presents inefficient computing power of the used numerical iterative calculations. Given the above, this paper introduces a Finite Element Analysis (FEA) process that is conducted to calculate the defect stresses of a boiler pressure steam pipeline from a certain power plant. This method combines the overall and partial pipeline modeling, which provides a new way of stress acquisition during the safety assessment of pressure steam pipeline containing defects.

\section{Structure Presentation of Pressure steam pipeline}

In a statutory periodical inspection process for a certain power plant, the boiler pressure steam pipeline as shown in Fig.1 was detected with two non-fusion defects of defect ZZQ-113 and PZQ-8, which laid a safety hazard for the normal operation of the pipeline. The working temperature and pressure of the pipeline are $400^{\circ} \mathrm{C}$ and 4.2MPa. According to the safety status level of the standard of TSG 21-2016"Supervision Regulation on Safety Technology for Stationary Pressure Vessel", two Defects are assessed as grade IV. In order to judge whether the pipeline can operate safely in the next statutory period, it is necessary to assess the fitness-forservice of the pipeline according to GB/T 19624-2019 "Safety Assessment of In-service Pressure Vessels Containing Defects". Forces and stress distributions at section position of pipeline defects, revealing the safe state of defects, are hard and key points in the safety assessment processes. Following study takes the stress

\footnotetext{
* Corresponding author: tangpingjob@163.com
} 
value of defect ZZQ-113 and PZQ-8 as the destination of calculations, aiming to describe universal finite element calculation method of defect stresses in pressure steam pipeline containing defects.

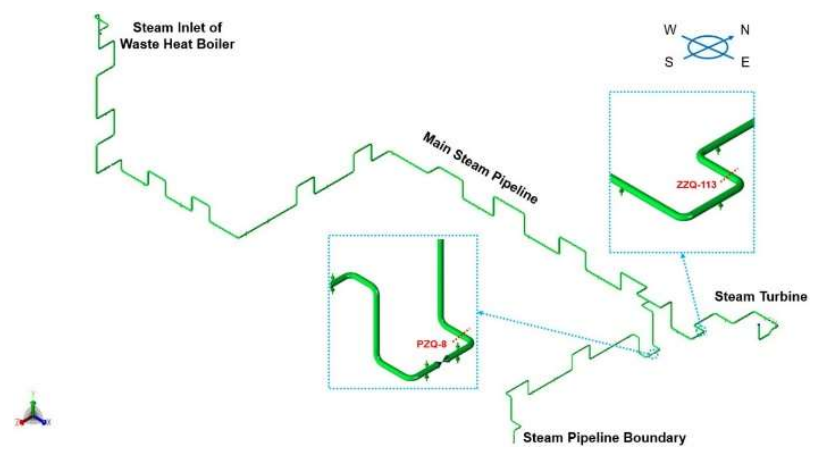

Figure 1. Pressure Steam Pipeline Geometry

\section{Solution Scheme of Pipeline Stress}

Given the difficulty of accomplishment of experimental measurement and theoretical calculation, the Finite Element Method (FEM) has become an available and effective way to obtain required data for researchers. There are two obvious shortcomings when used 3D FEM analysis ways are directly applied to solve defect stresses of pressure steam pipeline. The 3D FEM analysis model of a substantive pressure pipeline is full of a great number of grids that is inefficient for numerical iterative calculations, with higher CPU performance requirements. In addition, it is hard to involve complex factors such as rigid support load and self-adaptive spring hanger load of in-service pressure pipeline for common 3D FEA sofewares. CAESAR II developed by COADE company in the United States is the most widely used software of stress analysis and check for pressure pipeline in various industries. This tool fully takes into account the complex factors described above of in-service pressure pipeline. Its $1 \mathrm{D}$ beam element model consisted of simple nodes greatly improves the efficiency of iterative calculations. However, the stress data based on 1D beam element model is the section-averaged value in the node section location, which cannot express the most serious stress state in which defects are sensitive and vulnerable. Therefore, node stress solved by CAESAR II sofeware is powerless for the safety assessment of pressure pipeline containing defects.

Here, a method combines the overall and partial pipeline modeling is introduced for stress calculations of defects. There are two major contents of solution scheme. Firstly, it is needed to build pipline-wide $1 \mathrm{D}$ beam element CAESAR II calculation model of in-service assessed pressure pipeline in particular to set rigid support and selfadaptive spring hanger. The model makes full use of the advantages of CAESAR II software in modeling and calculating aspects, Comprehensively considering pipeline fittings, support constraints and external loads. A key point in this step is to empirically select and set the segmentation nodes based on locations of detected large- scale defects in the established pipline-wide model as the boundary of local defect part model. In iterative solutions, the force and moment of each node are calculated. The second content is to invoke 3D FEA commercial software to establish the shell element models of the local defect parts. Boundary conditions are imposed on these models equivalently from the above calculations described. Goal stress distributions of defects are presented by performing numerical iterative calculations by FEA solver where the principal stresses of defects are calculated based on the plastic strength level and the linear elastic theory of the material, with the assumption that there are no defects at detected defect locations of the pipeline. The solution process of goal stress is shown in Fig.2.

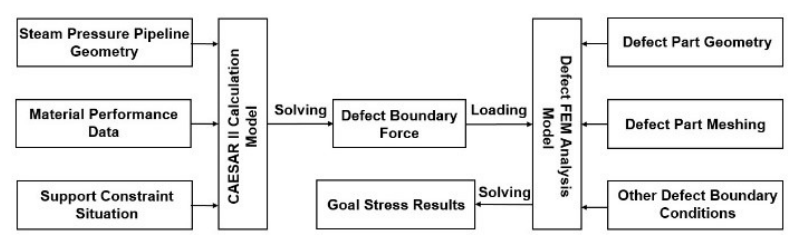

Figure 2. Defect Stress Solution Flowchart

Usually, the pipeline stress is non-linearly distributed over the thickness of pipeline. Goal stresses including the film stress $\sigma_{m}$ and the bending stress $\sigma_{B}$ used to assess pressure pipeline safety is the results of decomposing and calculating $\sigma_{1}$ and $\sigma_{2}$ according to formula (1). There $\sigma_{1}$ and $\sigma_{2}$ are the stress magnitudes on the inner and outer walls after stress linearization on the cross section of the defect, respectively, MPa. The stress linearization method of common surface defects is shown in Fig.3.

$$
\left\{\begin{array}{l}
\sigma_{m}=\left(\sigma_{1}+\sigma_{2}\right) / 2 \\
\sigma_{B}=\left(\sigma_{1}-\sigma_{2}\right) / 2
\end{array}\right.
$$
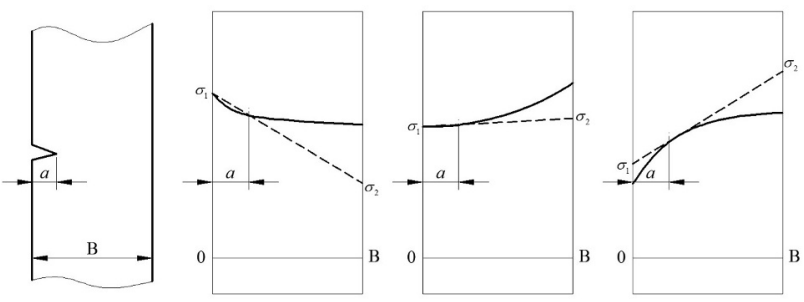

Figure 3. Stress Linearization Method of Surface Defects

\section{Efficient Program Code Design}

In some defect safety assessments of pressure steam pipelines containing defects, a number of FEA models of defect part pipeline may be needed to build, rather than only two defects in the current pressure steam pipeline. However, such work is repetitive, laborious and timeconsuming. And it does not mean that is simple or easy work. The modeling and meshing of 3D solid pipeline are very technical and highly experienced operation, especially for T-tubes, elbows and variable diameter tubes. The quality of meshing determines the accuracy and 
reliability of stress calculation results, which is the important guarantee for assessing pipeline safety. In order to save the time of building defect part models and ensure the grid quality of meshing defect part models, a program code in python language is designed. This code describes 3D graphics of pressure steam pipeline into computer language based on the mathematical algorithms of computer graphics, and realizes the stereo display of pressure steam pipeline in the user graphical interface. Then according to geometric topology, how to perfectly mesh pipeline model is conceived and accomplish by compiled code. Eventually, the building and meshing process of defect part models is automatically realized. Besides, the program code also has the function of imposing all boundary conditions including various types of support load. The graphical user interface developed is shown in Fig.4.

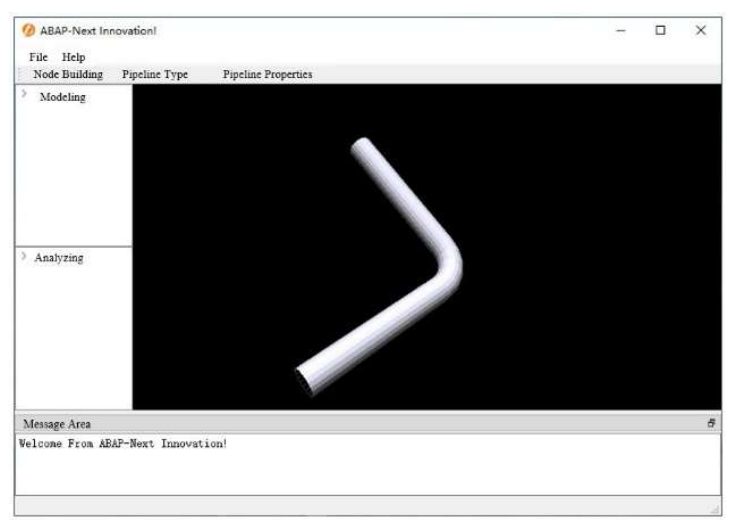

Figure 4. Graphical User Interface of Designed Program Code

All implementations of defect solution are operated in the graphical user interface. General input has following contents: the type of various pipeline segment, the coordinates of geometry nodes of line diagram, the outer diameter and the wall thickness, and so forth. Besides, the material and media properties of the pipeline and all the force boundary conditions are also necessary. Once the analyzing instruction is submitted, the designed program code directly and quickly invokes the FEA software installed on the computer to generate the 3D defect part model, to mesh the finite element model, to load the boundary conditions and to perform mechanical calculations in rapid sequence. The whole process runs automatically. The program code efficiently conducts complicated modeling and meshing of defect part geometry by a labor-saving way that just one-line nodes of pipelines are defined. All the formed grids are hexahedral structured grids with superior computational performance. The modeling and meshing process of the pressure steam pipeline defect ZZQ-113 is shown in Fig.5. With remarkable results, hours of work of defect analyses was completed in minutes with the designed efficient program code. Program code design above is truly an innovative highlight of the present stress calculation method.
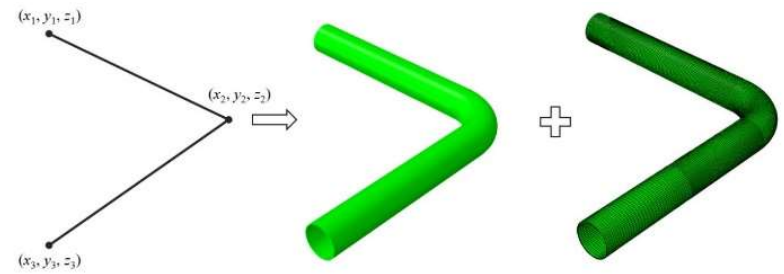

Figure 5. Efficient Modeling and meshing of Defect ZZQ-113

\section{Stress Calculation Results}

After the analyzing instruction was submitted, as results of iterative solutions, the stress distributions of defect part pipelines were presented in the form of a color cloud diagram. According to the stress distribution of defect ZZQ-113 as shown in Fig.6, the goal stresses of the defect were calculated through the stress linearization process performed on the cross-section of the defect. A stress path over wall thickness of pipeline was defined by picking start point and end point at which stress state was the most severe. Then the non-linear stress curves of dozens of nodes on this path were obtained. Linearize the stress curves in the way described above at the chapter of solution scheme of pipeline stress. Goal stresses both the film stress $\sigma_{m}$ and the bending stress $\sigma_{B}$ were calculated by substituding stress $\sigma_{1}$ and $\sigma_{2}$ on the inner and outer walls into formula (1). Finally, the film stress and the bending stress of the defect ZZQ-113 were $59.19 \mathrm{MPa}$ and 8.96 MPa, respectively. With the same treatment, the film stress of the defect PZQ-8 was $60.20 \mathrm{MPa}$; and the bending stress was $43.79 \mathrm{MPa}$.

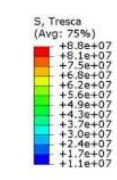

$$
{ }_{2}^{2}+x_{x}
$$
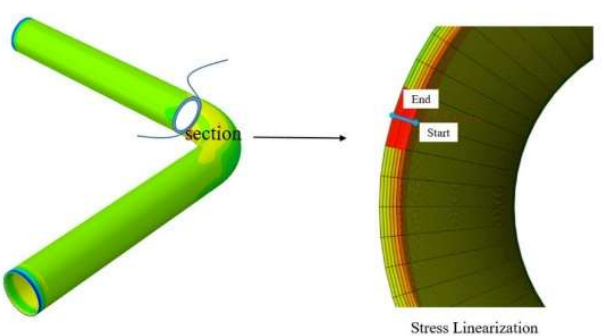

Figure 6. Stress Linearization of Defects ZZQ-113

\section{Conclusions}

The film stress and bend stress of two defects located on a pressure steam pipeline from a certain power plant were calculated in the present study. From this example, a combined FEA method of stress calculations was proposed. Follow this method, defect stresses are calculated with CAESRA II model and defect FEM analysis model in combination. Thereafter, an efficient program code with graphical user interface was designed to invokes the FEA software to automatically generate the 3D defect part model, to mesh the finite element model, to load the boundary conditions and to perform mechanical calculations in rapid sequence. In the current calculations, hours of work of defect analyses was completed in minutes with the designed code. That's a 
pleasant presentation of efficiency improvement. This paper provides an available way to obtain the stress state of the safety assessment of pressure steam pipeline containing defects.

\section{Acknowledgments}

This work was supported by Natural Science Foundation of Zhejiang Province (LQ21E050001) and the science and technology project of Zhejiang Provincial Administration for Market Regulation (CY2022221).

\section{References}

1. Pinheiro B, Soares C G, Pasqualino I. Generalized expressions for stress concentration factors of pipeline plain dents under cyclic internal pressure[J]. International Journal of Pressure Vessels and Piping, 2019, 170:82-91.

2. Witek M. Structural integrity of steel pipeline with clusters of corrosion defects[J]. Materials, 2021, 14(852):1-15.

3. Marti I, Sedmak A, Mitrovic N, et al. Effect of overpressure on pipeline structural integrity $[\mathrm{J}]$. Tehnicki Vjesnik, 2019, 26(3):852-855.

4. Dotta F, Ruggieri C. Structural integrity assessments of high pressure pipelines with axial flaws using a micromechanics model $[\mathrm{J}]$. International Journal of Pressure Vessels \& Piping, 2004, 81(9):761-770.

5. Adib-Ramezani H, Jeong J, Pluvinage G. Structural integrity evaluation of X52 gas pipes subjected to external corrosion defects using the SINTAP procedure[J]. International Journal of Pressure Vessels \& Piping, 2006, 83(6):420-432.

6. Kec J, Černý, Ivo, J Luštinec. Experimental verification of dents effect on the structural integrity of pipeline DN 300[J]. Key Engineering Materials, 2018, 774:319-324.

7. Yuanlong Lin, Zhenghong Yan. Application of CAESAR II in safety assessment of pressure pipe[J]. Petro-chemical Equipment, 2017(01):70-73. 